\title{
Computational intelligence for the detection and classification of malignant lesions in screening mammography
}

\author{
EVANGELIA PANOURGIAS $^{1}$, ATHANASIOS TSAKONAS ${ }^{2}$, GEORGIOS DOUNIAS $^{3}$ and GEORGIA PANAGI ${ }^{4}$ \\ ${ }^{1}$ Department of Radiology, Euroclinic Hospital, 9 Athanasiadou Str., 11521 Athens; ${ }^{2}$ Artificial Intelligence \\ and Information Analysis Laboratory, Department of Informatics, Aristotle University of Thessaloniki, \\ 54124 Thessaloniki; ${ }^{3}$ Department of Financial and Management Engineering, University of the Aegean, \\ 31 Fostini Street; ${ }^{4}$ Department of Radiology, General Hospital of Chios 'Skilitsion', 82100 Chios, Greece
}

Received September 6, 2005; Accepted September 28, 2005

\begin{abstract}
This report deals with the discussion of the findings obtained from the application of two computational intelligence methodologies for the detection of microcalcifications in screening mammography data. Genetic programming and inductive machine learning have been applied, in order to produce meaningful diagnostic rules for the medical staff. The data used in the experiments correspond to information acquired from two images of each breast of the patient, along with some associated patient information such as the age at time of study. Similar datasets have been previously used in an attempt to facilitate the development of computer algorithms to aid screening. Experienced screening radiologists have double-read the screening mammograms, they have weighted the malignancy ratings and averaged out the levels of suspiciousness assigned to each finding in the screenings. The diagnostic rules which were obtained from both genetic programming and machine learning have been evaluated in detail and then analyzed and discussed by collaborative medical experts, in parallel to findings from related literature. Results seem encouraging for further use and analysis by medical staff specializing in screening mammography.
\end{abstract}

\section{Introduction}

Breast cancer affects 1 of 9 women, $>180,000$ women are diagnosed with invasive cancer in the United States each year (1). Added to this, 25,000 cases of ductal and lobular cancer in situ are diagnosed annually. Breast cancer continues to be the leading cause of non-preventable cancer death in developed

Correspondence to: Dr Georgios Dounias, Department of Financial and Management Engineering, University of the Aegean, 31 Fostini Str., 82100 Chios, Greece

E-mail: g.dounias@aegean.gr

Key words: computational intelligence, computer assisted medical diagnosis, inductive machine learning, grammar guided genetic programming, screening mammography, microcalcifications countries. The early detection and diagnosis of breast cancer represents an important factor in its treatment and consequently the survival rate. Screening mammography is considered the most reliable method of early detection, accounting for a decrease in mortality of up to $18-23 \%$ (2). Although some cancers produce a mammographic appearance that is virtually pathognomonic of the disease, many malignant lesions have morphologic characteristics that cannot be differentiated from benign lesions. For this reason, biopsy of suspicious lesions plays an important role in the diagnosis of breast cancer. Breast cancer usually appears on a mammogram as: a) a spiculated mass (a dense, irregular, spiculated mass that is not related to prior surgery is the only feature that is virtually pathognomonic of a malignant lesion) $(3 ;$ p.377); b) pleiomorphic, heterogeneous microcalcifications (mammography is the only technique capable of detecting them). When found in isolation, microcalcifications are an indication of early-stage breast cancer (4); c) a focal asymmetric density with ill-defined margins or microlobulations, is a non-specific characteristic that suggests a malignant process; and d) an architectural distortion of the normal breast parenchyma which is a subtle manifestation of breast cancer.

The current work attempts to approach the problem of breast cancer diagnosis from screening mammography data using algorithmic approaches belonging to the field of artificial intelligence (AI). AI is considered the attempt of humans to equip computers with human logic and intelligence, when handling uncertain, approximate, or complex situations. Various methodologies for exploring this idea have been developed during the last decades, each representing a different viewpoint of the operating principles and cognitive mechanisms existing inside the human brain. Some of the most famous approaches of this kind (5) are: a) neural networks (an attempt to imitate mathematically, a simplified structure of the neuron-synapses network met inside the brain); b) fuzzy set theory (defines ways of handling ambiguity and quantifying or approximating fuzziness contained inside common language and reasoning); c) genetic algorithms and evolutionary computing (computational and mathematical representation of the main principles of Darwin's evolution theory, in the form of a computer algorithm); d) machine learning (based on the fact that, humans take decisions based on rational hypotheses and estimations - incorporated by mathematical 
logic and statistics - while they also add experience to their skills as they understand complex situations, by characterizing and classifying the importance of perceived information).

The present work, deals with two such computational intelligence approaches for medical diagnosis, namely inductive machine learning (6-8) and genetic programming (9-13). In computer literature a variety of intelligent approaches can be found trying to handle problems related to breast cancer diagnosis. In ref. 14 a combined approach of neural networks and evolutionary programming is presented, for computer assisted mammography. The work by Boccignone et al (15) proposes the use of information theory and wavelets for handling mammograms. The construction of a Bayesian network for mammographic diagnosis of breast cancer was proposed by Kahn et al (16). Mass lesion detection with a fuzzy neural network is attempted by Cheng and Cui (17). Purely neural network based approaches, the most popular on the matter of computational intelligence-based screening mammography diagnosis have been reported (18-23). An intelligent clustering method for the detection of microclassification clusters in mammography was presented (24). Fuzzy sets and pattern classification are combined for classification of microcalcifications (25). Finally, computer aided diagnosis approaches in mammograms have also been proposed by Sittek et al (26) and Christoyianni et al (27).

Below, we present the main computational intelligence methodological issues contained in this report, as well as details regarding the data set used to extract medical expert rules for screening mammography.

\section{Materials and methods}

We used data of 200 histologically proven malignant lesions discovered during screening to apply computer algorithms that may point in the direction of a specific histologic diagnosis. The data were collected during the last decade from the radiological departments of various Greek hospitals. All available data were inserted in a two dimensional matrix, where each column represented one diagnostic attribute (the last one denoting the final diagnosis for the training cases) and each row represented the complete diagnostic information regarding each case collected. Data were either numerical or nominal. Their analysis was performed using algorithms based on inductive machine learning and genetic programming, aiming at producing a set of rules in an attempt to differentiate between the various types of breast cancers using mammography and pathology data.

Among different ML approaches, entropy information based inductive machine learning has been the most widely and successfully applied tool $(6,7)$. The idea is based on the measurement of entropy information when coping with historical (past) data, as well as the 'divide and conquer' principle for forming inductive decision trees, proposed theoretically by Hunt et al (8). Inductive machine learning based on measures of entropy information is capable of handling medical diagnosis in practice, because of the nature of problems met in medical decision making, such as: a) difficulties in applying mathematical modelling techniques, b) existence of nominal attributes and approximate quantities and concepts, c) difficulties in discrimination among various
Table I. Diagnostic attributes of the study.

Age
Mass-shape and size (where applicable)
Mammographic parenchymal pattern
Presence of microcalcifications
Presence of architectural distortion
Other associated findings (nipple retraction/skin thickening)
BIRADS score (1-5)
Histologic diagnosis
Lymph node status
Estrogen receptor status
Progesterone status
Histologic size

possible diagnoses, d) heuristic nature of the solutions applied, and e) possible missing attribute values, etc. In the last decade, ML theories have also served as the basis for the spread of modern methodologies used in addition to classical statistics, for advanced data analysis in complex domains of application.

On the other hand, genetic programming operates by mimicking a living population where survival of the fittest applies. Fitness is how successful a member is in completing a task and the least fit members are eliminated. Also, new members are added by means of mutation, breeding and random generation. Regarding the genetic optimization approach followed within this work, we construct rule-bases using genetic programming (GP) $(9,28)$ techniques. The idea is addressed in refs. 9,29 where the genetic programming approach is used to build a kind of decision trees. Genetic programming, found with a proper function set, has been proved capable of finding optimal solutions in a reasonable time for a variety of classification tasks (29). Genetic programming is an extension to the inspiration of genetic algorithms (GA) (30). The GP theory enables the use of functional tree-nodes that offer powerful intelligent tools like the symbolic regression problem solving (29). An extension to the concept of standard GP is the strongly typed or type-constrained GP $(10,31)$. By using the latter approach, it is possible to construct two-valued logic (modus ponens) expressions with the use of grammars, which preserve a satisfactory rate of success when used as classification rules in many domains $(28,31)$.

For each case of breast cancer, all four standard mammographic views were used (craniocaudal and mediolateral oblique, for each breast), as well as clinical and pathology data. All cases were rated according to the level of concern by using the standard Breast Imaging Reporting and Data System, or BIRADS, recommendations. BIRADS consists of a lexicon of terminology that aims to standardize mammography reporting. A coding system has been designed to facilitate database maintenance (32). A total of 12 diagnostic attributes shown in Table I, were given as an input into the algorithm. Brief comments follow, regarding the attributes of the study. 
Firstly, all women included in the study were $>45$ years old. Regarding, mammographic parenchymal pattern, there is a classification system devised by Tabar that categorizes the breast parenchyma into subtypes. In each of these patterns, the mammographic image is a mixture of the structural components of the breast, i.e. lobules, fibrous tissue, adipose tissue, milk ducts and blood vessels. Pattern I refers to relatively dense breast tissue, most commonly found in premenopausal women. Pattern II and III represent the endresult of involution, where the images are dominated by adipose tissue. Pattern IV refers to dense, nodular breast tissue where perception of small lesions is often difficult. Pattern $\mathrm{V}$ is dominated by extensive fibrosis and the capability of mammography to reveal small pathologic lesions, is limited (33). The attribute referred in Table I as other associated findings, includes the presentce of nipple retraction and skin thickening. Finally, regarding BIRADS score (1-5), category I refers to a negative mammogram and category II includes all mammograms with a benign finding. Mammograms of these two categories require no further evaluation and were not included in the study. Mammograms classified as BIRADS III means that a finding of intermediate concern was detected that requires further evaluation. Category IV includes mammograms with lesions that have a high probability of malignancy, such as microcalcifications and category $\mathrm{V}$ refers to mammograms with a lesion that is probably malignant, such as a spiculated mass.

The main histologic types of breast cancer include ductal cancer DCIS (ductal cancer in situ) and invasive ductal cancer, and lobular cancer LCIS (lobular cancer in situ) and invasive lobular cancer. Over $80 \%$ are variants of ductal cancer. In the non-invasive type (DCIS) the tumor cells are confined to the duct epithelium and do not penetrate the basement membrane, whereas in the invasive type, the tumor cells invade the basement epithelium and invade the surrounding tissues. Mammography has made it possible to detect DCISs, and they comprise $20-30 \%$ of detected cancers in a screening program.

The majority of invasive cancers are invasive ductal cancers and account for $65 \%$ of the breast cancers diagnosed in the United States (34). Non-invasive lobular carcinoma, lobular cancer in situ or LCIS, does not form a palpable mass or a visible lesion by mammography, so they were not included in the study. A woman with LCIS has a $15 \%$ chance of developing an invasive cancer in either breast, with a total risk of $30 \%$ over the next 20 years and beyond (35). For this reason, it is currently classified as a premalignant lesion rather than a true cancer.

Invasive lobular cancer frequently infiltrates the normal breast tissue in a diffuse manner, without causing architectural distortion. This may be the reason why it is not often apparent on mammograms and is generally larger at diagnosis. Invasive lobular cancer accounts for $<10 \%$ of breast cancer, it tends to be bilateral more often than ductal cancer $(20 \%$ of the cases are bilateral) and also, multicentric in the same breast (36).

All mammograms were reviewed and the imaging attributes of the mammograms were recorded. The imaging attributes included the presence of: a) a mass, its shape (spiculated, round, oval) and its margins (ill-defined or well-defined), b) microcalcifications and their shape (pleiomorphic, hetero- geneous), c) architectural distortion, and d) associated findings (nipple retraction and skin retraction). Breast lesion location defined as quadrant (upper outer, lower outer, upper inner, lower inner) and left or right breast were also noted. The pathology and other data were recorded on a separate sheet, so the radiologist was initially blinded to the histology diagnosis.

\section{Results}

Although GP obtains higher diagnostic accuracy and better generalization over the available dataset, it came out that ML generally performs superior to GP, in terms of comprehensibility and medical impact, from the purely medical viewpoint. Below we give the most interesting extracted rules obtained with the two computational intelligence approaches applied on the specific screening mammography data.

If a mass with ill-defined margins is observed in the right breast, in the upper outer quadrant, it is most likely invasive ductal cancer. It is a well known fact that the presence of an ill-defined or spiculated mass is observed on a mammogram, it is almost pathognomonic of an invasive ductal cancer. This type of malignancy produces collagen and causes a desmoplastic reaction and fibrosis. This produces a spiculated mass on a mammogram (3). Also, most cancers (60-70\%) are observed in the upper outer half of the breast.

If a patient presents with a focal asymmetric density and a BIRADS score 3 in the right breast, the lesion is suggestive of invasive ductal cancer, if the size is $>14 \mathrm{~mm}$ or in both breasts. Invasive lobular cancer cells have decreased E-cadherin expression which is a glue-like substance that provides cell-to-cell adhesion, a feature prominent in invasive ductal carcinoma that causes cells to stick together and produce a mammographically visible mass (37). This is why invasive lobular cancer is less apparent on mammograms and therefore, generally larger at diagnosis. Silverstein et al found that the average size at diagnosis for invasive ductal cancers was $23 \mathrm{~mm}$ and for invasive lobular cancers $30 \mathrm{~mm} \mathrm{(38).}$

If a patient presents a suspicious lesion and a BIRADS score 5 in the upper inner quadrant of the right breast and the size of the lesion is $<21 \mathrm{~mm}$, then the diagnosis is most probably invasive ductal cancer, whereas if it is $>21 \mathrm{~mm}$ then it is invasive lobular cancer.

If a patient presents a focal asymmetric density, a BIRADS score 5 and a lesion size $<42 \mathrm{~mm}$, then the diagnosis is more often invasive ductal cancer, whereas if it is $>42 \mathrm{~mm}$, then it is invasive lobular cancer. A mammogram with a suspicious lesion that has been classified as category $\mathrm{V}$ of BIRADS score suggests a high probability of malignancy and often is due to the presence of an invasive ductal cancer. It is interesting to note that in a study that included 50,000 invasive ductal cancers and invasive lobular cancers, Arpino et al found that $54 \%$ of invasive lobular cancers are $>2 \mathrm{~cm}$, compared to $48 \%$ of invasive ductal cancers (39). It was also found that invasive lobular cancers presented as a large tumor exceeding $5 \mathrm{~cm}$, as compared with $9 \%$ of invasive ductal cancers. 
Table II. Risk of breast cancer in relation to age.

\begin{tabular}{lc}
\hline Age & Cancer per 100,000 women per year \\
\hline $25-29$ & 7.4 \\
$30-34$ & 26.7 \\
$35-39$ & 66.2 \\
$40-44$ & 129.4 \\
$45-49$ & 159.4 \\
$50-54$ & 220.0 \\
$55-59$ & 261.6 \\
$60-64$ & 330.7 \\
$65-69$ & 390.7 \\
$70-74$ & 421.8 \\
$75-79$ & 461.4 \\
\hline
\end{tabular}

Source: based on SEER data 1984-1988 National Institutes of Health. National Cancer Institute Statistics Review 1975-1988. Bethesda, MD: National Institutes of Health; 1991. NIH Publication no. 912789.

If a patient with a BIRADS score 4 presents with suspicious microcalcifications on a mammogram in the upper outer quadrant and an associated architectural distortion, then the diagnosis is more likely to be invasive ductal cancer, whereas if the microcalcifications are not accompanied by architectural distortion, then the diagnosis of DCIS is more probable. DCIS is a form of malignant transformation of the epithelial cells lining the mammary ducts and lobules. The proliferating cells are confined by an intact basement membrane. Necrotic debris in the lumen of the duct produces microcalcifications which are visible on a mammogram (40). Breaks in the basement membrane result in stromal invasion of cancer cells, causing invasive ductal cancer which is visible on a mammogram.

If a mass is $\geq 3$, meaning if it is obscured or spiculated then the histology diagnosis is invasive ductal cancer. A spiculated lesion on a mammogram is virtually pathognomonic of breast cancer. Strands of fibro-malignant tissues, radiating out from an ill-defined mass, produces this spiculated appearance ( 3 ; p.580). Many cancers do not elicit the desmoplasia that produces spiculation, but even in these cases, tumor infiltration is often reflected in the mammographically ill-defined or, less often, in partly obscured borders. However, a well-circumscribed benign lesion may seem to have obscured margins because of superimposing breast tissue. This often results in the need for ultrasound or tissue sampling to differentiate the lesion.

If the mass margin is $<3$, i.e. circumscribed, ill-defined or lobulated, and the size is $<1 \mathrm{~cm}$, then the lesion is invasive ductal cancer, otherwise if it is $>1 \mathrm{~cm}$, then it is invasive lobular cancer. The values of the variables for mass margins correspond to ' 0 = circumscribed', ' 1 = ill-defined', ' 2 = lobulated', '3 = obscured', ' 4 = speculated'. Generally, circumscribed or lobulated lesions are more often benign masses and only $7 \%$ of malignant lesions are well-circum- scribed and usually the medullary type which have a more favorable diagnosis (41), or rarer types of malignancies such as lymphoma, colloid and papillary carcinoma. Ill-defined margins suggest a malignant process, because they are believed to represent tumor infiltration of the surrounding tissue.

\section{Discussion}

The aforementioned results suggest several interesting trends. Despite the limited information (no prior studies, no normal cases, many more cases of invasive ductal cancer than other types of cancer) and the fact that different types of abnormalities (microcalcifications, masses, architectural distortion) were included, the classification performances of determining that an identified lesion was a specific histologic subtype was reasonable and consistent when using inductive machine learning techniques. Note that the initial experimentation did not produce the expected results about the role of age as a criterion for separating between different types of breast cancer diagnosis, due to the fact that the subjects used in the study were rather not representatively selected for the entire value range. For this reason, age was later excluded from further experimentation. Nevertheless, according to literature (Table II) it is known that the risk of developing breast cancer increases with age, with a rapid rise in incidence after the age of 50, a fact which is in tune with our main experimental finding pointing the critical value of 54 years of age for indicating the presence of a type of breast cancer. Another fact coming up from our study is that the extracted rules often included the right breast as a determining factor. This warrants further investigation as it has not been proven in the literature. At this point, it should be noted that there are many other data useful for breast cancer diagnosis that were not used here, such as: a) prior mammographic studies for comparison, b) inclusion of other types of cancer besides invasive ductal cancer that comprised the majority of our case, and c) benign tumoral lesions. This is probably the reason why the performance of genetic programming classification systems was generally below expectations (most of the rules presented in this paper came from the inductive machine learning approach). The computerized classification methods often used histology findings such as size to categorize the mammographic lesions. It would be interesting to see how well the classification systems would perform without histology data, using only a larger number of radiological features. These issues have to be further investigated with larger datasets that include a greater number of attributes, a substantial amount of normal patients and more cases of cancers other than invasive ductal cancers that composed the majority of our present dataset to improve the performance of the classification systems, particularly ones relying of genetic programming. There is great potential for improvement, but ultimately, the most significant test in the future will be to assess where radiologists can use such classification systems to improve their clinical performance.

\section{References}

1. Ries LAG, Eisner MP, Kosary CL, Hankey BF, Miller BA, Clegg L, et al (eds): SEER Cancer Statistics Review. National Cancer Institute, Bethesda, MD, pp1973-1999, 2002. 
2. Tabar L, Yen MF, Vitak B, Chen HH, Smith RA and Duffy SW: Mammography service screening and mortality in breast cancer patients: 20-year follow-up before and after introduction of screening. Lancet 361: 1405-1410, 2003.

3. Kopans DB: Breast Imaging. 2nd edition. Lippincott-Raven Publishers, Philadelphia, 1998.

4. Stomper PC, Connolly JL, Meyer JE and Harris JR: Clinically occult ductal carcinoma in situ detected with mammography: analysis of 100 cases with radiologic and pathologic correlation. Radiology 172: 235-241, 1989.

5. Mitchell TM: Machine Learning. McGraw-Hill Publ. Corp., New York, 1997.

6. Quinlan JR: Induction of decision trees. Machine Learning 1: 81, 1986.

7. Quinlan JR: C4.5 Programs for Machine Learning. Morgan Kauffman Publishers, London, 1993.

8. Hunt EB, Marin J and Stone PJ: Experiments in Induction. Academic Press, New York, 1966.

9. Koza JR: Genetic Programming - On the Programming of Computers by Means of Natural Selection. MIT Press, 1992.

10. Montana DJ: Strongly typed genetic programming. Evol Comput 3: 2,1995

11. Hussain T: Cellular Encoding: Review and Critique. Technical Report, Queen's University, http://www.qucis.queensu ca/home/ hussain/web/1997_cellular_encoding_review.ps.gz, 1997.

12. Ratle A and Sebag M: Genetic Programming and Domain Knowledge: Beyond the Limitations of Grammar-Guided Machine Discovery. Schienauer et al (eds). Proceedings of the 6th Conference on Parallel Problems Solving from Nature, LNCS. Springer, Berlin, pp211-220, 2000.

13. Singleton A: Genetic programming with $\mathrm{C}++$. BYTE Magazine: 2, 1994 .

14. Fogel DB, Wasson EC, Boughtonand EM and Porto VW: A step toward computer-assisted mammography using evolutionary programming and neural networks. Cancer Lett 119: 93-97, 1997.

15. Boccignone G, Chianese A and Picariello A: Using Renyi's information and wavelets for target detection: an application to mammograms. Pattern Anal Appl 3: 303-313, 2000.

16. Kahn CE Jr, Roberts LM, Shaffer KA and Haddawy P: Construction of a Bayesian network for mammographic diagnosis of breast cancer. Comp Biol Med 27: 19-29, 1997.

17. Cheng HD and Cui M: Mass lesion detection with a fuzzy neural network. Pattern Recognition 37: 1189-1200, 2004.

18. Lauria A, Palmiero R, Forni G, Cerello P, Golosio B, Fauci F, Magro R, Raso G, Tangaro S and Indovina PL: The CALMA system: an artificial neural network method for detecting masses and microcalcifications in digitized mammograms. Nuclear Instruments and Methods in Physics Research Section A: Accelerators, Spectrometers, Detectors and Associated Equipment 518: 391-393, 2004.

19. Papadopoulos A, Fotiadis DI and Likas A: An automatic microcalcification detection system based on a hybrid neural network classifier. Artif Intell Med 25: 149-167, 2002.

20. Lucht R, Delorme S and Brix G: Neural network-based segmentation of dynamic MR mammographic images. Magn Reson Imaging 20: 147-154, 2002.

21. Qian W, Sun X, Song D and Clark RA: Digital mammography: wavelet transform and Kalman-filtering neural network in mass segmentation and detection. Acad Radiol 8: 1074-1082, 2001.
22. Markopoulos C, Kouskos E, Koufopoulos K, Kyriakou V and Gogas J: Use of artificial neural networks (computer analysis) in the diagnosis of microcalcifications on mammography. Eur $\mathbf{J}$ Radiol 39: 60-65, 2001.

23. Tsujii O, Freedman MT and Mun SK: Classification of microcalcifications in digital mammograms using trend-oriented radial basis function neural network. Pattern Recognition 32: 891-903, 1999.

24. Zhang L, Sankar R and Qian W: Advances in micro-calcification clusters detection in mammography. Comput Biol Med 32: 515-528, 2002.

25. Grohman WM and Dhawan AP: Fuzzy convex set-based pattern classification for analysis of mammographic microcalcifications. Pattern Recognition 34: 1469-1482, 2001.

26. Sittek H, Herrmann K, Perlet C, Künzer I, Kessler M and Reiser M: Computer-aided diagnosis in mammography. Radiologe 37: 610-616, 1997.

27. Christoyianni I, Koutras A, Dermatas E and Kokkinakis G: Computer aided diagnosis of breast cancer in digitized mammograms. Comput Med Imaging Graph 26: 309-319, 2002.

28. Wong ML: A flexible knowledge discovery system using genetic programming and logic grammars. Decision Support Systems 31: 405-428, 2001.

29. Koza JR, Bennett FH III, Andre D and Keane MA: Genetic Programming III. Morgan Kaufmann, 1999.

30. Goldberg DE: Genetic Algorithms in Search, Optimization and Machine Learning. Addison-Wesley, Reading, MA, 1989.

31. Gruau F: Using Syntactic constraints with genetic programming In: Advances in Genetic Programming. Angeline PJ and Jinnear KE Jr (eds). MIT, 1996.

32. D'Orsi CJ: The American College of Radiology Breast Imaging Reporting and Data System. 2nd edition. Reston VA, American College of Radiology, 1995.

33. Gram IT, Funkhouser E and Tabar L: The Tabar classification of mammographic parenchymal patterns. Eur J Radiol 24: 131-136, 1997.

34. Stalsberg $\mathrm{H}$ and Thomas DB: Age distribution of histologic types of breast cancer. Int J Cancer 54: 1-7, 1993.

35. Wheeler JE, Enterline HT, Roseman JM, et al: Lobular carcinoma in situ of the breast: long-term follow-up of the breast. Cancer 34: 554-563, 1974.

36. Dixon JM, Anderson TJ, Page DL, et al: Infiltrating lobular cancer of the breast: incidence and consequence of bilateral disease. Br J Surg 70: 513-516, 1983.

37. Moll R, Mitze M, Frixen UH and Birchmeier W: Differential loss of E-cadherin expression in infiltrating ductal and lobular breast carcinomas. Am J Pathol 143: 1731-1734, 2004.

38. Silverstein MJ, Lewinsky BS, Waisman JR, et al: Infiltrating lobular carcnoma: it is different from infiltrating duct carcinoma? Cancer 73: 1673-1677, 1994.

39. Arpino G, Bardou VJ, Clark GM and Richard M: Elledge infiltrating lobular carcinoma of the breast: tumor characteristics and clinical outcome. Breast Cancer Res 6: 149-156, 2004.

40. Holland R and Hendriks JHCL: Microcalcifications associated with ductal carcinoma in situ: mammographic-pathologic correlation. Semin Diagn Pathol 11: 181-192, 1994.

41. Meyer JM, Amin E, Lindfors KK, et al: Medullary carcinoma of the breast: mammographic and sonographic features. Radiology 170: 79-82, 1989. 